\title{
Cadherins as Predictive Markers of Nodal Metastasis in Breast Cancer
}

Maya Madhavan, M.Sc., Priya Srinivas, M.Sc., Elizabeth Abraham, M.D., Iqbal Ahmed, M.S., Aleyamma Mathew, Ph.D., N. R. Vijayalekshmi, Ph.D., Prabha Balaram, Ph.D.

Division of Cancer Research (MM, PS, PB), Department of Pathology (EA), Department of Surgical Oncology (IA), Department of Epidemiology (AM), Regional Cancer Centre; and Department of Biochemistry, University of Kerala (NRV), Trivandrum, India

Adhesion molecules, particularly cadherins play a pivotal role in cancer invasion and metastasis. Because the therapeutic management of tumors with and without nodal metastasis differs considerably, our idea was to identify tumors with metastatic potential. We studied the expression of E-cadherin and P-cadherin immunohistochemically in 51 cases of breast cancer that included 29 node-negative and 22 node-positive cases. Expression of the cadherins was mainly membranous, with cytoplasmic staining in a few lesions. Both E-cadherin and P-cadherin showed significant down-regulation of their expression in node-positive tumors in comparison to node-negative tumors. Logistic regression analysis revealed that the positive expression of E-cadherin and P-cadherin showed low odds ratios of 0.1 and 0.2 , respectively, and were statistically significant. On multivariate analysis, both the cadherins were found to be of independent prognostic value. This suggests that cadherin expression could be a marker of nodal metastasis. An observation of interest was that the expression of E-cadherin and $P$-cadherin were highly correlated (correlation coefficient $=0.5873$ ), which requires further evaluation for confirmation of a common regulatory pathway that could be activated in the early onset of nodal metastasis.

KEY WORDS: E-cadherin, Immunohistochemistry Nodal metastasis, P-cadherin.

Mod Pathol 2001;14(5):423-427

Copyright ( 2001 by The United States and Canadian Academy of Pathology, Inc.

VOL. 14, NO. 5, P. 423, 2001 Printed in the U.S.A.

Date of acceptance: December 22, 2000

This work was supported in part by the Department of Science, Technology and Environment, Government of Kerala, India. MM is a recipient of a junior research fellowship from the Council of Scientific and Industrial Research, Government of India.

Address reprint requests to: Dr. Prabha Balaram, Regional Cancer Centre, Trivandrum, Kerala, India 695 011; e-mail: rcctvm@md2.vsnl.net.in; fax: 91-0471-447454.
Metastasis poses the most serious threat to clinicians in the management of breast cancers. The problem attains even greater dimension because of the different therapeutic modalities adopted in cases with and without nodal metastasis. The process of metastasis, either locoregionally into lymph nodes or systemically into distant organs, remains intriguing, despite various studies in this field. As an initial step in metastasis, tumor cells should detach from the primary site and enter the circulation. This is thought to be due to changes in the cell-cell adhesive properties. Hence, adhesion molecules are supposed to play an important part in cancer invasion.

Cadherins deserve particular attention among the recently identified group of invasion suppressor genes (1). Cadherins are $\mathrm{Ca}^{2+}$-dependent adhesion molecules that mediate homophilic cell-cell adhesion (2). The cadherin gene family encompasses E-cadherin, P-cadherin, and $N$-cadherin. A number of studies have found an inverse correlation between the expression of E-cadherin and the tumor stage in carcinomas of breast (3) and prostate (4), squamous cell carcinoma of head and neck (5), and so on. Graff et al. (6) found that the reduced expression of E-cadherin is due to hypermethylation of the $\mathrm{CpG}$ island of the promoter region of the E-cadherin gene. In the present study, we attempted to correlate the expression of cadherins with nodal metastasis in carcinomas of the breast.

\section{MATERIALS AND METHODS}

\section{Patients and Tissue Specimens}

Formalin-fixed, paraffin-embedded blocks of 51 randomly selected tumor specimens from primary breast cancer patients previously classified as node negative or node positive were retrieved from the files. The study group included 29 node-negative and 22 node-positive patients. The medical records of these patients were reviewed, and clinical details were collected. 


\section{Antibodies}

Goat polyclonal antibodies against E-cadherin $(\mathrm{N}-20)$ and P-cadherin (N-19) of Santa Cruz Biotechnology (Santa Cruz, CA) were used at 1:20 dilution. All the dilutions were done in $1 \times$ phosphate buffered saline (PBS).

\section{Immunohistochemistry}

The standard avidin-biotin indirect immunoperoxidase method (Santa Cruz Biotechnology kit) was used for immunohistochemistry. Briefly, $4-\mu \mathrm{m}$ sections were cut from the paraffin embedded blocks using a microtome. The glass slides were previously coated with poly-L-lysine. The sections were then incubated at $37^{\circ} \mathrm{C}$ overnight. Thereafter, the sections were deparaffinised in xylene (30 minutes, twice), sequentially dehydrated by incubating in 1:1 xylene-alcohol mixture, $100 \%$ alcohol, $90 \%$ alcohol, $70 \%$ alcohol, $50 \%$ alcohol, $30 \%$ alcohol and $1 \times$ PBS (10 minutes each). Antigen unmasking by autoclaving was done in $0.01 \mathrm{~m}$ citrate buffer ( $\mathrm{pH}$ 6.0) for 5 min. After cooling and washing, the endogenous peroxidase of erythrocytes was blocked by incubating in $30 \% \mathrm{H}_{2} \mathrm{O}_{2}$ for 30 minutes to prevent nonspecific binding of antibodies, the sections were incubated with $3 \%$ BSA for 30 minutes. Thence, the sections were incubated overnight with the primary antibodies, washed, incubated with biotinylated secondary antibodies (30 minutes), washed, and incubated with avidin-peroxidase complex (30 minutes). The peroxidase reaction was visualized by incubating with AEC for 10 minutes in the dark. All washings were done in $1 \times$ PBS.

\section{Evaluation of Immunostaining}

Sections were examined under a light microscope. The staining pattern was assessed without the prior knowledge of histopathological diagnosis. The normal breast tissues adjacent to the tumor areas served as an internal control. The scoring was as follows: negative, 1; mild positive, 2; moderate positive, 3; intense positive, 4 . The staining pattern was evaluated by an independent investigator $(\mathrm{PB})$ and confirmed in consultation with the pathologist (EA). The staining score was expressed as mean value \pm SE. The staining pattern in both E-cadherin and P-cadherin were almost similar and is shown in Figure 1, A-D.

\section{Statistical Analysis}

Association between the antigen expression and nodal status was evaluated by rank correlation using Mann-Whitney $U$ Wilcoxon Rank Sum test for two independent samples. Using a logistic regression model, multivariate analysis was done, and odds ratios were estimated to assess whether cad- herins could serve as a marker for developing node positivity. $P$ values $<.05$ were considered statistically significant.

\section{RESULTS}

\section{E-cadherin Expression}

The expression of E-cadherin was localized uniformly to the cell membrane with intense staining at the intercellular junctions. A few samples showed loss of strictly membranous expression pattern but showed cytoplasmic expression. There was a significant difference in the expression of E-cadherin between those tumors with and without nodal metastases $(P=.009$; Table 1 , Table 2$)$. The expression of E-cadherin was significantly downregulated in higher grade tumors $(P=.028$; Table 3$)$. The odds ratio was 0.1 for E-cadherin expression in nodepositive patients compared with unity in nodenegative patients, calculated at $95 \%$ confidence interval $(P=.001$; Table 4$)$.

\section{P-Cadherin Expression}

The staining pattern of P-cadherin was almost similar to that of E-cadherin, with localization of expression to cell membrane. There was a significant down-regulation of P-cadherin in nodepositive breast cancers, with a $P$ value of .008 (Table $3)$. Though not significantly, the expression of P-cadherin was higher in low-grade tumors in comparison to high-grade tumors (Tables 1 and 2). The odds ratio was 0.2 for P-cadherin-positive patients, whereas it was unity for P-cadherin-negative patients $(P=.05)$ (Table 4$)$.

Using a logistic regression model, multivariate analysis was done using all the classical variables (stage, grade, tumor type, proliferation rate assessed by PCNA staining, menstrual status) and the cadherins. Of these variables, excluding tumor type and grade of tumor, all the other variables were found to be independent prognostic predictors of nodal positivity.

Looking at the bivariate correlations, we find that apart from the cadherins being significantly correlated to the nodal status, E-cadherin and P-cadherin also show a significant bivariate correlation between them (Table 3). On the whole, in $47 \%$ of the cases, both the cadherins were reduced or absent, whereas in $37 \%$ of the cases, both of them were expressed.

\section{DISCUSSION}

Cadherins have been proposed to play an important role in activating the mechanism of tumor dissemination from the primary site. They are an important member of the recently identified group 

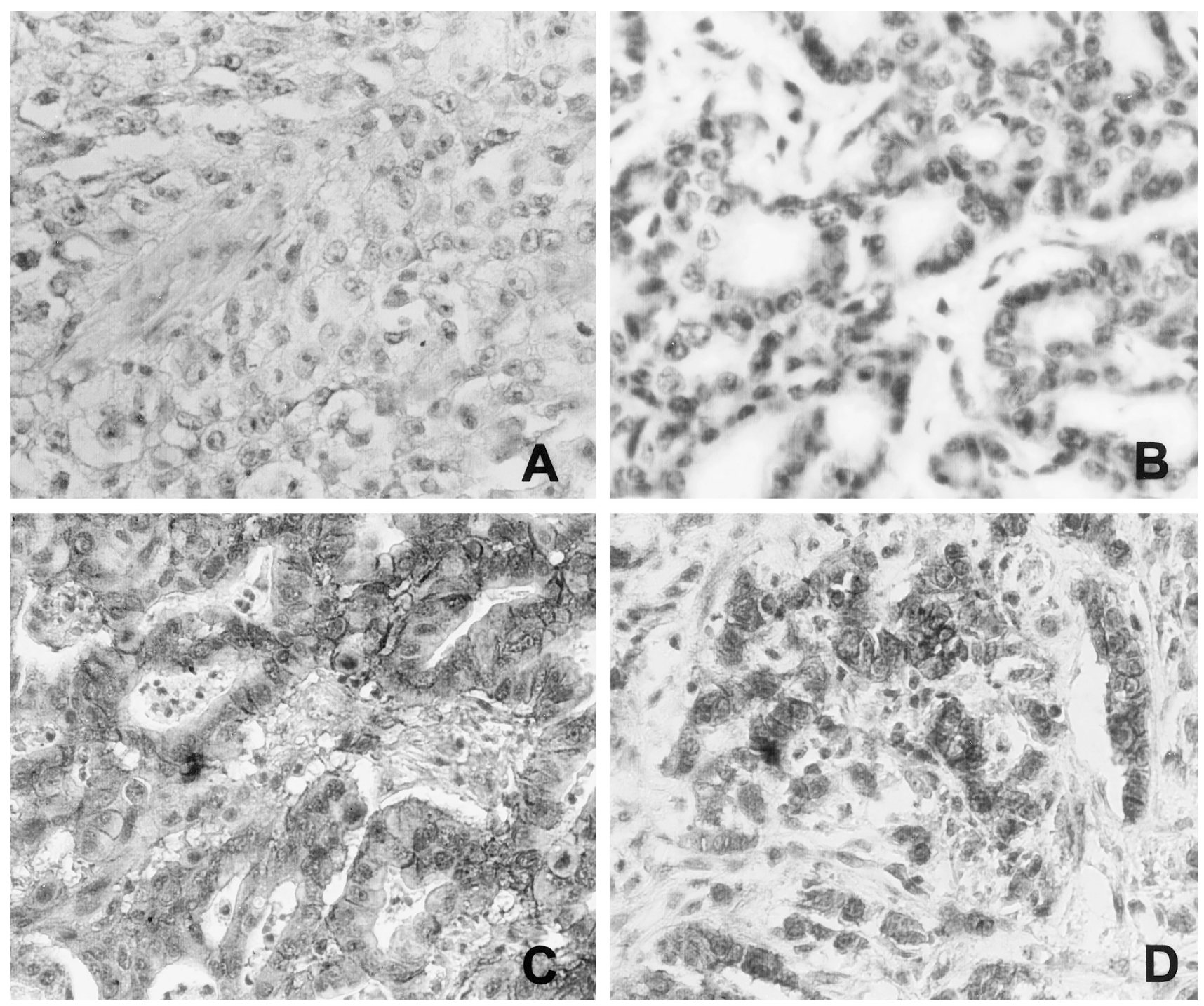

FIGURE 1. Microphotograph of breast cancer tissue showing staining pattern of cadherins (E-cadherin and P-cadherin). (A) Negative staining. (B) Mild membranous staining. (C) Moderate membranous staining. (D) Intense membranous staining.

TABLE 1. Expression of E-Cadherin and P-Cadherin in Breast Cancer

\begin{tabular}{cccc}
\hline \multirow{2}{*}{ Protein } & \multicolumn{2}{c}{ Mean Score \pm SE } & \multirow{2}{*}{$P$ Value } \\
\cline { 2 - 3 } & Node - ve & Node + ve & \\
\hline E-cadherin & $28966 \pm 0.167$ & $1.2273 \pm 0.091$ & .009 \\
P-cadherin & $2.6897 \pm 0.173$ & $1.4545 \pm 0.171$ & .364 \\
\hline
\end{tabular}

of metastasis suppressor genes. In the present study, we used immunohistochemistry because it facilitates the identification of the protein localization and a semiquantitation of the protein. The results of the study demonstrate that the expression of cadherins is lower in carcinomas of the breast that are node positive than in those that are node negative. E-cadherin has been used as an immunohistochemical tool for the diagnosis and prognosis of various carcinomas (7). Umbas et al. (8) reported a decreased expression of E-cadherin as indicative of a poor clinical course of the disease. The transi- tion from well-differentiated adenoma to invasive carcinoma in mouse pancreatic $\beta$-cell carcinogenesis has been found to be due to loss of E-cadherin expression (9). The potential for E-cadherin to function as an invasion suppressor in epithelial tumorigenesis has been supported by in vitro studies. E-cadherin-negative epithelial cells grow invasive with a mesenchymal phenotype. After transfection with E-cadherin cDNA, epithelial structure is restored (10). The loss of membranous staining pattern in some of our samples could mean the presence of a nonfunctional E-cadherin, as suggested by Gagliardi et al. (11). Diminution of cadherin expression is postulated to remove the regulatory control of contact inhibition on proliferation, thus allowing escape from growth control signals (12). Pece et al. (13) have shown that E-cadherin can initiate an outside-in signal-transducing pathway that regulates the activity of phosphoinositide-3kinase and Akt, thus providing a novel mechanism 
TABLE 2. Distribution of Staining Intensity of Cadherins According to Nodal Status

\begin{tabular}{|c|c|c|c|c|c|c|c|c|}
\hline \multirow{3}{*}{$\begin{array}{l}\text { Nodal } \\
\text { Status }\end{array}$} & \multicolumn{8}{|c|}{ Distribution of Staining Intensity (\%) } \\
\hline & \multicolumn{4}{|c|}{ E-cadherin } & \multicolumn{4}{|c|}{ P-cadherin } \\
\hline & Negative & Mild & Mode rate & Intense & Negative & Mild & Mode rate & Intense \\
\hline Negative & 17.4 & 13 & 56.5 & 13 & 17.4 & 17.4 & 34.8 & 30.4 \\
\hline Positive & 50 & 29.2 & 20.8 & 0 & 48 & 32 & 8 & 12 \\
\hline
\end{tabular}

TABLE 3. Relation of Cadherin Expression with Nodal Status and Grade of Tumor

\begin{tabular}{lcc}
\hline \multicolumn{1}{c}{ Variables } & $\begin{array}{c}\text { Rank Correlation } \\
\text { Coefficient }\end{array}$ & $P$ Value \\
\hline E-cadherin/node & -0.5436 & .001 \\
P-cadherin/node & -0.3701 & .008 \\
E-cadherin/grade & -0.3670 & .028 \\
P-cadherin/grade & -0.2301 & .177 \\
E-cadherin/P-cadherin & 0.5873 & .001 \\
\hline
\end{tabular}

TABLE 4. Analysis of Cadherins and Other Variables as Risk Factors for Node Positivity

\begin{tabular}{lccc}
\hline \multicolumn{1}{c}{ Variable } & $\begin{array}{c}\text { Regression } \\
\text { Coefficient }\end{array}$ & Odds Ratio & P Value \\
\hline Stage & 1.4267 & 4.1648 & .001 \\
Proliferation rate & 1.7578 & 5.7998 & .006 \\
Menstrual status & 0.6951 & 2.000 & .040 \\
E-cadherin & -2.2254 & 0.1000 & .001 \\
P-cadherin & -1.6864 & 0.2000 & .001 \\
\hline
\end{tabular}

whereby the intercellular adhesion may control the fate of the epithelial cells. This study shows a downregulation of the cadherins in node-positive tumors, and at multivariate analysis, cadherins were found to be of independent prognostic value in predicting the nodal status. We found that alterations in the expression of cadherins offer a useful adjunct to current prognostic indicators. This is also evidence for the significant inverse correlation of E-cadherin with grade of the tumor. Thus, measurement of cadherin expression, in concert with other markers of differentiation, may provide an additional prognostic tool.

What leads to down-regulation of cadherins upon metastasis, be it locoregional or distant, is still not clearly defined. It is proven that calcium influxinduced proteolysis of E-cadherin not only disrupts the cell-cell adhesion but also activates intracellular signaling pathway, potentially leading to alterations in motility and proliferation activity of cells. Calcium influx activates a membrane-bound metalloprotease that induces degradation of the ectodomain of E-cadherin (14). Graff et al. (15) reported that the cadherin that was downregulated in the initial stage of metastasis should get reexpressed in the metastatic deposits.

Analysis of odds ratio of the cadherins showed that their down-regulation can serve as predictive markers of nodal metastasis in breast cancers. Taken together, the cadherins could be better indi- cators of nodal metastasis. This suggests a crucial role for the expression of cadherins in demarcating node-positive from node-negative cases. This is very significant in patients presenting with a primary tumor and histologically undiagnosable micrometastasis. At the same time, subjecting nodenegative patients with palpable nodes to higher courses of treatment can be avoided. We suggest that development and use of cadherins as diagnostic markers could solve this problem and could be an additional tool for physicians in the effective therapeutic management of such tumors.

Another important result is a significant correlation between the cadherins. This could mean a common regulatory pathway for the cadherins that gets activated upon initiation of metastasis. This putative pathway could be crucial in deciding the metastatic fate of a tumor. Elucidation of this pathway may help in the therapeutic design of antimetastatic drugs. Further investigations are necessary to examine the molecular mechanism underlying the regulation of cadherins.

\section{REFERENCES}

1. Behrens J, Frixen U, Schipper J. Cell adhesion in cancer invasion and metastasis. Semin Cancer Biol 1992;3:169-178.

2. Takeichi M. Cadherins: a molecular family important in selective cell-cell adhesion. Annu Rev Biochem 1990;59:23752.

3. Oka HS, Shiozaki H, Kobayashi K, Inoue M, Tahara H, Kobayashi $\mathrm{T}$, et al. Expression of E-cadherin cell adhesion molecules in human breast cancer tissues and its relationship to metastasis. Cancer Res 1993;53:1696-701.

4. De Marzo AM, Knudsen B, Chan-Tack K, Epstein JI. E-cadherin expression as a marker of tumor aggressiveness in routinely processed radical prostatectomy specimens. Urology 1999;53:707-13.

5. Schipper JH, Frixen UH, Behrens J, Unger A, Jahnke K, Birchmeier W. E-cadherin expression in squamous cell carcinomas of head and neck; inverse correlation with tumor dedifferentiation and lymph node metastasis. Cancer Res 1991; 51:6328-37.

6. Graff JR, Herman JG, Lapidus RG, Chopra H, Xu R, Jarard DF, et al. E-cadherin expression is silenced by DNA hypermethylation in human breast and prostate carcinomas. Cancer Res 1995;55:5195-99.

7. Birchmeier W, Behrens J. Cadherin expression in carcinomas: role in the formation of junctions and the prevention of invasiveness. Biochim Biophys Acta 1994;198:11-26.

8. Umbas R, Isaacs WB, Bringuier PP, Schaafsma HE, Karthaus HFM, Oosterhof GON, et al. Decreased E-cadherin expression is associated with poor prognosis in patients with prostate cancer. Cancer Res 1994;54:3929-33. 
9. Perl AK, Wilgenbus P, Dahl U, Semb H, Christofori G. A causal role for E-cadherin in the transition from adenoma to carcinoma. Nature 1998;392:190-3.

10. Frixen UH, Behrens J, Sachs M, Eberle G, Voss B, Warda A, et al. E-cadherin mediated cell-cell adhesion prevents invasiveness of human carcinoma cells. J Cell Biol 1991;113:17385.

11. Gagliardi G, Kandemir O, Liu D, Guida M, Benvestito S, Ruers TGM. Changes in E-cadherin immunoreactivity in the adenoma-carcinoma sequence of the large bowel. Virchows Archiv 1995;426:149-54.

12. St. Croix B, Sheehan C, Rak JW, Florenes VA, Slingisland JM, Kerbel RS. E-cadherin dependent growth suppression is mediated by the cyclin dependent kinase inhibitor p27 KIPI. J Cell Biol 1998;142:557-71.
13. Pece S, Chiariello M, Murga C, Gutkind JS. Activation of the protein kinase Akt/PKB by the formation of E-cadherin mediated cell-cell junctions. Evidence for the association of phosphoinositide-3-kinase with the E-cadherin adhesion complex. J Biol Chem 1999;274:19347-51.

14. Ito K, Okamoto T, Araki N, Kawano Y, Nakao M, Fujiyama S, et al. Calcium influx triggers the sequential proteolysis of extracellular and cytoplasmic domains of E-cadherin, leading to loss of $\beta$-catenin from cell-cell contacts. Oncogene 1999;18:7080-90.

15. Graff JR, Gabrielson E, Fujii H, Baylin SB, Herman JG. Methylation patterns of the E-cadherin $5^{\prime} \mathrm{CpG}$ island are unstable and reflect the dynamic, heterogeneous loss of E-cadherin expression during metastatic progression. J Biol Chem 2000; 275:2727-32. 